\title{
S1 Leitlinie - Österreichischer Konsensus zur Antikoagulation im Rahmen der Vorhofflimmerablation
}

\author{
Martin Martinek · Marianne Gwechenberger · Daniel Scherr • Clemens Steinwender • Markus Stühlinger • \\ Helmut Pürerfellner · Franz Xaver Roithinger · Lukas Fiedler · Arbeitsgruppe Rhythmologie der Österreichischen \\ Kardiologischen Gesellschaft
}

Eingegangen: 29. Oktober 2017 / Angenommen: 21. Dezember 2017 / Online publiziert: 25. Januar 2018 (c) Der/die Autor(en) 2018. Dieser Artikel ist eine Open-Access-Publikation.

\begin{abstract}
Zusammenfassung Zusammenfassend kann eine ununterbrochene orale Antikoagulation mit unterschiedlichen Empfehlungsklassen und Evidenzlevel sowohl für die VKA- als auch für die NOAC-Therapie im Rahmen der Pulmonalvenenisolation empfohlen werden. Selbst bei niedrigen $\mathrm{CHA}_{2} \mathrm{DS}_{2}$-VASc-Werten ist 3-4 Wochen vor und 8 Wochen nach dem Eingriff eine OAK indiziert. Eine periinterventionelle Überbrückung mit Heparinen sollte aufgrund erhöhter Blutungsereignisse vermieden werden. Der vorliegende Konsens gibt Empfehlungen zum aktuellen Kenntnisstand und wurde ausschließlich von Mitgliedern der Arbeitsgruppe Rhythmologie der Öster-
\end{abstract}

PD Dr. M. Martinek, FESC, FHRS .

Prof. Dr. H. Pürerfellner, FESC, FEHRA

Abteilung für Innere Medizin 2 mit Kardiologie, Angiologie und Internistischer Intensivmedizin, Ordensklinikum Linz, Elisabethinen, Fadingerstraße 1, 4020 Linz, Österreich martin.martinek@ordensklinikum.at

ao.Univ. Prof. Mag. phil. Dr. med. M. Gwechenberger ( $₫)$ Abteilung für Kardiologie, Universitätsklinik für Innere Medizin II, Wien, Österreich

marianne.gwechenberger@meduniwien.ac.at

Assoz.Prof. PD Dr. D. Scherr, FESC, FEHRA

Universitätsklinik für Innere Medizin, Klinische Abteilung für Kardiologie, Medizinische Universität Graz, Graz, Österreich

Prim. PD Dr. C. Steinwender, FESC

Klinik für Kardiologie und Internistische Intensivmedizin, Kepler Universitätsklinikum Linz, Medizinische Fakultät der Johannes Kepler Universität, Linz, Österreich

PD Dr. M. Stühlinger

Kardiologie, Universitätsklinik für Innere Medizin III, Innsbruck, Österreich

Prim. Univ. Doz. Dr. F. X. Roithinger, FESC · OA Dr. L. Fiedler 2. medizinische Abteilung für Innere Medizin,

Landesklinikum Wiener Neustadt, Wien, Österreich reichischen Gesellschaft für Kardiologie erstellt, die über große praktische Erfahrungen in der Katheterablation und periinterventionellen OAK bei Patienten mit Vorhofflimmern verfügen. Die Veröffentlichung neuer randomisierter und kontrollierter Studien zu diesem Thema wird in den kommenden Monaten erwartet, so dass es sicherlich Änderungen in den Empfehlungen geben wird. Die Arbeitsgruppe für Rhythmologie der österreichischen Kardiologischen Gesellschaft wird sich bemühen, diese S1-Richtlinie regelmäßig auf dem neuesten Stand zu halten. Wir hoffen, dass dieser Konsens dazu genutzt wird, die Sicherheit für Patienten, die einer Pulmonalvenenisolation unterzogen werden, zu erhöhen und den behandelnden Ärzten einen homogenen Ansatz in Österreich zu ermöglichen.

Schlüsselwörter Ablation • Vorhofflimmern · Vitamin K Antagonisten (VKA) · Nicht-Vitamin K orale Antikoagulantien (NOAC) $\cdot$ Consensus

S1 guideline - Austrian consensus for anticoagulation in the context of atrial fibrillation ablation

Summary In summary, uninterrupted oral antikoagulation can be recommended, with different recommendation classes and levels of evidence, for both, VKA and NOAC therapy, in the framework of PVI. Even with low $\mathrm{CHA}_{2} \mathrm{DS}_{2}$ VASc scores, OAK is indicated 3-4 weeks before and 8 weeks after the procedure. Periinterventional bridging with heparins should be avoided due to increased bleeding events.

The present Consensus provides recommendations on the current state of knowledge and has been prepared exclusively by members of the Rhythmology Working Group of the Austrian Cardiological Society who have great practical experience in catheter ab- 
lation and peri-interventional OAK in patients with atrial fibrillation. Publication of new randomized and controlled studies on the subject are expected in the coming months, so that there will certainly be changes in the recommendations. The Rhythmology Working Group of the Austrian Cardiological Society will strive to keep this S1 guideline regularly up to date. We hope that this consensus is used to increase the safety for patients undergoing PVI and to provide physicians with a homogeneous approach in Austria.

Keywords Ablation - Atrial fibrillation · Vitamin K antagonists (VKA) · Non-vitamin k oral anticoagulants $(\mathrm{NOAC}) \cdot$ Consensus

\section{Einleitung}

Patienten, die unter symptomatischem Vorhofflimmern (VHF) leiden und einer linksatrialen Ablation (in diesem Dokument generell als Pulmonalvenenisolation, PVI bezeichnet, auch wenn zusätzliche Ablationen durchgeführt werden) unterzogen werden, haben häufig einen erhöhten $\mathrm{CHA}_{2} \mathrm{DS}_{2}$-VASc-Score und damit ein erhöhtes Schlaganfallrisiko. Insbesondere vor, während und nach einer linksatrialen Ablation ist die adäquate Antikoagulation zur Verhinderung systemischer Thrombembolien unverzichtbar. Aufgrund der steigenden Datenlage gegenüber den Vitamin $\mathrm{K}$ Antagonisten (VKA), gewinnen auch die nicht-Vi- tamin $\mathrm{K}$ oralen Antikoagulantien (NOAC) in diesem klinischen Zusammenhang an Bedeutung. NOAC weisen gegenüber VKA Vorteile in der klinischen Handhabung auf, die einen schnellen Wirkungseintritt, kurze Halbwertszeiten, die fehlende Notwendigkeit von regelmäßigen Gerinnungskontrollen aufgrund größerer therapeutischer Breite und weniger Medikamenteninteraktionen beinhalten. Bisher bestand in der Anwendung der NOAC im Rahmen der PVI eine uneinheitliche Vorgehensweise. Die aktuellen Empfehlungen basieren auf dem 2017 HRS/EHRA/ ECAS/APHRS/SOLAECE Expert Consensus Statement für Katheter- und Chirurgische Ablation von Vorhofflimmern, den ESC Guidelines $2016 \mathrm{zu}$ VHF, beziehungsweise auf praktische Empfehlungen der ERHA (European Heart Rhythm Association) aus 2015 und 2017 [1, 2]. Bei allen Empfehlungen (Tab. 1), werden auch die dahinterliegenden Publikationen angegeben.

Die PVI wurde bei selektierten Patienten mit VHF inzwischen als Standardtherapie etabliert [3, 6]. Obwohl die orale Antikoagulation erst bei Patienten mit VHF und einem $\mathrm{CHA}_{2} \mathrm{DS}_{2}$-VASc Score $\geq 2$ (Männer) bzw. $\geq 3$ (Frauen) eine Klasse-I Indikation darstellt, sind de facto viele Patienten mit niedrigerem $\mathrm{CHA}_{2} \mathrm{DS}_{2}$-VASc Score bereits vor der Ablation oral antikoaguliert [2]. Grundsätzlich wäre in diesem Fall vor der Ablation eine orale Antikoagulation (OAK) für 3-4 Wochen oder ein linksatrialer Thrombenausschluss mittels transösophagealer Echokardiographie

Tab. 1 Consensus-Empfehlungen zur Antikoagulation im Rahmen der Pulmonalvenenisolation

\begin{tabular}{|c|c|c|c|}
\hline Consensus-Empfehlungen & Klasse & Grad & Ref \\
\hline $\begin{array}{l}\text { Alle Patienten sollten nach einer PVI zumindest für } 8 \text { Wochen weiter antikoaguliert werden (unabhängig vom } \\
\mathrm{CHA}_{2} \mathrm{DS}_{2} \text {-VASC-Score). }\end{array}$ & $\mathrm{a}^{\mathrm{a}}$ & C & {$[1-5]$} \\
\hline $\begin{array}{l}\text { Die Antikoagulation sollte auch nach einer erfolgreichen PVI bei Patienten mit hohem Schlaganfallrisiko } \\
\text { (CHA } \mathrm{DS}_{2} \text {-VASC-Score) weitergeführt werden. }\end{array}$ & $\mathrm{I}^{\mathrm{b}}$ & C & {$[1-3,6]$} \\
\hline Die OAK mit VKA soll im Rahmen der PVI ununterbrochen weitergeführt werden (INR 2-3). & $I^{\mathrm{C}}$ & A & {$[1,2,7,8]$} \\
\hline Die OAK mit Dabigatran soll im Rahmen der PVI ununterbrochen weitergeführt werden. & I & B & {$[1,9-17]$} \\
\hline Die OAK mit Rivaroxaban soll im Rahmen der PVI ununterbrochen weitergeführt werden (präferentiell Abendgabe). & I & B & {$[1,2,18,19]$} \\
\hline Die OAK mit Apixaban soll im Rahmen der PVI ununterbrochen weitergeführt werden. & Ila & B & {$[1,2,20-25]$} \\
\hline Die OAK mit Edoxaban soll im Rahmen der PVI ununterbrochen weitergeführt werden. & lla & C & {$[1,2,26]$} \\
\hline $\begin{array}{l}\text { Periinterventionelles Bridging mit niedermolekularen Heparinen soll (bei zuvor bereits antikoagulierten Patienten) } \\
\text { vermieden werden. }\end{array}$ & III & B & {$[2,8]$} \\
\hline Intraprozedural wird im Rahmen der PVI die Gabe von unfraktioniertem Heparin mit einer ACT über 300 s empfohlen. & 1 & B & {$[1,2,23,27-29]$} \\
\hline $\begin{array}{l}\text { Bei Patienten, die vor der PVI nicht therapeutisch antikoaguliert waren und auf VKA eingestellt werden, ist ein } \\
\text { Bridging mit niedermolekularem (s. c.) oder unfraktioniertem (i. v.) Heparin bis zum Erreichen eines therapeutischen } \\
\text { INR notwendig. }\end{array}$ & 1 & C & {$[1,2]$} \\
\hline $\begin{array}{l}\text { Bei Patienten, die vor der PVI nicht therapeutisch antikoaguliert waren und auf NOAC eingestellt werden, ist die } \\
\text { NOAC-Gabe 3-5h nach dem Erreichen der Hämostase sinnvoll (ein Bridging mit Heparin ist nicht indiziert). }\end{array}$ & lla & C & {$[1,30-35]$} \\
\hline $\begin{array}{l}\text { Bei Patienten, die vor der PVI auf ein NOAC eingestellt sind, kann die morgendliche NOAC Dosis vor der Ablation } \\
\text { pausiert werden. }\end{array}$ & lla & B & {$[1,30-35]$} \\
\hline $\begin{array}{l}\text { Die Durchführung einer transösophagealen Echokardiographie ist bei Patienten sinnvoll, die sich zur PVI im persistie- } \\
\text { renden VHF präsentieren, auch wenn sie für zumindest } 3 \text { Wochen vorher therapeutisch antikoaguliert waren. }\end{array}$ & lla & C & {$[1,2,36]$} \\
\hline $\begin{array}{l}\text { Die Durchführung einer transösophagealen Echokardiographie ist bei Patienten sinnvoll, die vor der PVI nicht für } \\
\text { zumindest } 3 \text { Wochen therapeutisch antikoaguliert waren. }\end{array}$ & lla & C & {$[1,2,36]$} \\
\hline \multicolumn{4}{|c|}{$\begin{array}{l}\text { Klasse Evidenzklasse, Grad Evidenzgrad, Ref Referenzen, PVI Pulmonalvenenisolation bzw. linksatriale Ablation, auch wenn zusätzliche Ablationen (z. B.: Linien, } \\
\text { etc.) durchgeführt werden, OAK orale Antikoagulation, VKA Vitamin K Antagonist, ACT activated clotting time, NOAC Nicht-Vitamin K orales Antikoagulans } \\
\text { aLt. ESC Ila, It. HRS Consensus I } \\
\text { bLt. ESC Klasse lla, jedoch bei nicht-PVI Patienten I A. Lt. HRS I } \\
\text { 'Lt. ESC Ila, It. HRS I }\end{array}$} \\
\hline
\end{tabular}


indiziert. Die OAK mit VKA vor, während und nach einer PVI sollte mit einem therapeutischen INR von 2-3 eingestellt sein. Die Prozedur selbst beinhaltet jedoch ein relevantes Risiko sowohl für thrombembolische (Insult bis $1 \%$, stummen zerebralen Läsionen; SZL 5-20\%) als auch für Blutungskomplikationen (Leistenkomplikationen nach Punktion 1,5\%, Perikardtamponade und Hämatothorax 1-2\%)[4]. Diesbezüglich konnten sowohl randomisierte als auch Registerstudien zeigen, dass die ununterbrochene Gabe von VKA sicherer ist als die überbrückende Therapie mit unfraktioniertem oder niedermolekularem Heparin, sodass ein Bridging nicht mehr durchgeführt werden sollte $[7,8]$.

In der Re-Circuit-Studie konnte für die ununterbrochene Gabe von Dabigatran während der PVI eine Reduktion von Blutungen gegenüber VKA gezeigt werden, thrombembolische Ereignisse waren in beiden Gruppen sehr selten [9]. Die durchgehende Gabe von Rivaroxaban während der PVI wurde im Rahmen der Venture AF Studie untersucht. Auch diese Strategie zeigte vergleichbare Eventraten zu ununterbrochener Gabe eines VKA [18]. Die bisher lediglich als Abstract präsentierte AEIOU Studie fand bei Apixaban (in zwei verschiedenen Dosierungen) eine ähnliche Effektivität und Sicherheit, wie der VKA [20]. Randomisierte Studien zur ununterbrochenen Gabe der Faktor Xa Antagonisten Apixaban und Endoxaban sind zu Beginn 2018 zu erwarten.

Dieses Consensus Dokument der Arbeitsgruppe für Rhythmologie der österreichischen Kardiologischen Gesellschaft wurde durch die Zusammenarbeit der Autoren entwickelt und unter den AG Mitgliedern für weitere Kommentare zirkuliert, um einen möglichst breite Übereinstimmung zu erhalten. Dieses Consensus-Dokument stellt eine Leitlinie zum derzeitigen Wissensstand zur Anwendung OAK im Rahmen der Ablation von VHF dar und soll ein homogenes Vorgehen in ganz Österreich ermöglichen.

Wie bei den ESC Guidelines wurden die ConsensusEmpfehlungen in Empfehlungsklassen und Evidenzlevel eingeordnet [2]:

Eine Klasse I Empfehlung bedeutet, dass es Evidenz und/oder generelle Übereinstimmung darüber gibt, dass eine Behandlung günstig, nützlich und effektiv ist und somit durchgeführt werden soll bzw. indiziert ist („SOLL“-Empfehlung).

Bei einer Klasse II Empfehlung gibt es widersprüchliche Evidenz und/oder divergierende Meinungen über den Nutzen oder die Wirksamkeit einer Behandlung. Eine Klasse IIa Empfehlung wird ausgesprochen, wenn die Evidenz und/oder Meinung für eine Behandlung überwiegt („SOLLTE“-Empfehlung). Bei einer Klasse IIb Empfehlung ist der Nutzen oder die Wirksamkeit weniger gut untermauert („KANN“Empfehlung).

Eine Klasse III Empfehlung wird ausgesprochen, wenn es Evidenz und/oder generelle Übereinstimmung darüber gibt, dass eine Behandlung nicht güns- tig, nützlich oder effektiv ist oder sogar schädlich sein könnte (nicht indiziert/kontraindiziert).

Ebenso erfolgt die Einteilung der Evidenzlevels nach den ESC Guidelines in [2]:

Level of Evidence (LOE) A, wenn Daten mehrerer randomisierter und kontrollierter Studien (RCT) oder Meta-Analysen vorliegen; $L O E B$, wenn eine einzelne RCT oder große non-RCTs vorliegen; $L O E C$, wenn lediglich Expertenmeinungen, Daten kleinerer Studien, retrospektiver Studien oder aus Registern vorliegen.

\section{Vitamin K Antagonisten}

Wegen des verzögerten Wirkungseintritts und -abfalls wurden VKA in der Vergangenheit vielfach "gebridged“, d.h. der VKA wurde in den Tagen vor der Ablation abgesetzt und durch niedermolekulares Heparin ersetzt. In der COMPARE Studie konnte erstmalig gezeigt werden, dass dieses Vorgehen im Rahmen einer PVI gegenüber einer ununterbrochenen Gabe von VKAs mit einem durchgehend bestehenden INR von 2-3 mit einer erhöhten Blutungs- und Schlaganfallrate behaftet ist [8]. Eine rezente Metaanalyse von Nairooz et al. konnte die Überlegenheit einer durchgehenden Gabe von VKA (Ziel-INR 2-3) gegenüber dem Heparin-Bridging hinsichtlich Endpunkten wie Schlaganfall oder Blutung bestätigen [37]. Somit ist im Falle einer periprozeduralen Gabe eines VKA in jedem Fall die ununterbrochene Gabe angezeigt, was in den rezenten Guidelines der ESC zum VHF 2016 mit einer IIa-Indikation und in HRS/EHRA/ APHRS/ECAS/SOLAECE Consensus-Papier 2017 mit einer Klasse I Indikation versehen ist. Die OAK sollte (auch bei niedrigem $\mathrm{CHA}_{2} \mathrm{DS}_{2-}$ VASc Score $\leq 1$ ) zumindest 3-4 Wochen vor dem Eingriff begonnen werden, alternativ kann eine transösophageale Echokardiographie erfolgen $[1,2]$.

Die bereits erwähnte RE-CIRCUIT Studie untersuchte Blutungs- und Schlaganfallraten bei Patienten im Rahmen einer PVI unter VKA vs. Dabigatran. Die Rate an schweren Blutungen war im VKA Arm mit 6,9\% ungewöhnlich hoch und nicht im Bereich sonstiger zur ununterbrochenen VKA Therapie durchgeführten Studien [9]. Eine rezente Metaanalyse von $\mathrm{Wu}$ et al. mit mehr als 11.000 Patienten, die einer PVI unterzogen wurden, zeigte keinen Unterschied in der Rate an Schlaganfällen oder schweren Blutungen beim periprozeduralen Einsatz von NOAC gegenüber dem Einsatz von VKA [38].

Insgesamt stellt somit der ununterbrochene Einsatz von VKA mit einem Ziel INR von 2-3 eine sowohl durch Studiendaten als auch durch Guidelines abgesicherte Therapieoption bei Patienten im Rahmen der PVI dar.

\section{Dabigatran}

Dabigatran ist ein direkter Thrombininhibitor und war das erste NOAC, das auf den Markt gekommen 
ist. Die Substanz wurde in der RELY-Studie an über 18.000 Patienten mit nicht-valvulärem VHF in 2 Dosierungen getestet. Beide Dosierungen waren Warfarin hinsichtlich des primären Endpunkts (Schlaganfall und systemischer Embolie) nicht unterlegen. Die höhere Dosierung mit Dabigatran $150 \mathrm{mg} 2 \times$ täglich war dem Warfarin hinsichtlich dem Auftreten von Schlaganfall und systemischer Embolie überlegen (RR 0.65, $95 \%$ CI: $0.52-0.81 ; p<0,001)$ und konnte als einziges NOAC das Auftreten eines ischämischen Schlaganfalls, bei gleicher Blutungsrate, gegenüber Warfarin reduzieren (HR 0.75; $95 \% \mathrm{CI}$ : 0.58-0.97; $p=0,03$ ). Darüber hinaus fand sich eine Reduktion der vaskulären Mortalität, eine Reduktion der Gesamtmortalität wurde knapp nicht erreicht. Die niedrigere Dosierung mit 110 mg $2 \times$ täglich war gleich effektiv wie Warfarin hinsichtlich ischämischer Insulte und systemischer Embolien. Das Auftreten schwerer und lebensbedrohlicher Blutungen wurde signifikant reduziert, die intrakranielle Hämorrhagie war in beiden Dosierungen signifikant geringer [39]. Diese positiven Ergebnisse konnten in mehreren Datenbankenanalysen („Real world“ Daten) bestätigt werden und zeigen sich auch bei Anwendung über längere Zeit konstant [40-44].

Mit Idarucizumab steht das erste spezifische und derzeit einzig zugelassene Antidot für ein NOAC zu Verfügung. Idarucizumab ist ein monoklonales, humanisiertes Antikörperfragment und bindet mit einer 350-mal höheren Affinität an die Thrombin-bindende Stelle von Dabigatran als an Thrombin. Dadurch hebt Idarucizumab die gerinnungshemmende Wirkung von Dabigatran sehr rasch und vollständig auf, wie in Studien mit gesunden Probanden und bei Patienten mit milder bis moderater Niereninsuffizienz gezeigt werden konnte [10, 11]. In der REVERSE-AD wurde Idarucizumab an Patienten untersucht, die eine unkontrollierbare bzw. lebensbedrohende Blutung hatten oder eine $(n)$ dringende $(n)$ Intervention bzw. chirurgischen Eingriff benötigten. Sie konnte zeigen, dass die Gerinnungshemmung nach Gabe von Idarucizumab innerhalb von Minuten aufgehoben werden kann. Bisher gibt es keinen Hinweis auf Nebenwirkungen im Sinne von immunogenen oder prothrombotischen Effekten [10-13].

Zahlreiche Studien konnten die Sicherheit und Effektivität von Dabigatran im Rahmen von Ablationen zeigen [14-16, 45-51]. Diese wurden auch im Rahmen einer Meta-Analyse bestätigt [17]. Allerdings waren die meisten nur Beobachtungsstudien und die Gabe von Dabigatran wurde unterschiedlich lange vor dem Eingriff pausiert. Die RE-CIRCUIT Studie untersuchte prospektiv die ununterbrochene Gabe von Dabigatran $(2 \times 150 \mathrm{mg})$ im Vergleich zu ununterbrochenem Warfarin (INR 2-3) bei 704 Patienten, die sich einer PVI unterzogen. Der primäre Endpunkt war das Auftreten von schwerwiegenden Blutungen. In der Dabigatran Gruppe zeigten sich signifikant weniger schwere Blutungen $(5[1,6 \%]$ vs. 22 [6,9\%] Ereignisse; relative Risikoreduktion $77 \%$; absolute Ri- sikoreduktion 5,3\%). In der Studie wurde lediglich ein thrombembolisches Ereignis in der Warfarin-Gruppe beobachtet. Einschränkend muss gesagt werden, dass die Studie ohne Nullhypothese geplant wurde, da aufgrund der niedrigen Inzidenz der Ereignisse zu viele Patienten eingeschlossen werden müssten und daher nur explorativen Charakter hat. Trotzdem erscheint durch zunehmende Fallzahlen und Evidenz eine ununterbrochene Gabe von Dabigatran sicher und effektiv [9].

\section{Rivaroxaban}

In der ROCKET-AF Studie war der Faktor Xa-Antagonist Rivaroxaban einem VKA (Warfarin) in der Vermeidung von Schlaganfällen und systemischen Embolien bei Patienten mit nicht-valvulärem VHF nicht unterlegen. Es bestand auch kein signifikanter Unterschied in Hinblick auf das Risiko schwerwiegender Blutungen, wobei intrakranielle und tödliche Blutungen unter Rivaroxaban jedoch seltener auftraten [52].

Für das NOAC Rivaroxaban liegen bei der PVI im Vergleich zu VKA die Ergebnisse einer kleinen, jedoch randomisierten Studie (VENTURE-AF) sowie mehrerer Register, zusammengefasst in einer Meta-Analyse von Vamos et al., vor [18, 19]. Die Meta-Analyse detektierte dabei keine relevanten Unterschiede in Hinblick auf Effektivität und Sicherheit zwischen Rivaroxaban und VKA.

Da sowohl aus Gründen der Praktikabilität als auch der Sicherheit während der Katheterablation eine ununterbrochene bzw. nicht-,gebridgte“ OAK-Gabe von besonderem Interesse ist, sollen hier die Ergebnisse der VENTURE-AF Studie, die in diesem Setting die ununterbrochene Gabe von Rivaroxaban und VKA verglich, beleuchtet und als Leitfaden verwendet werden [18]:

Es wurden 248 typische Kandidaten für eine Katheterablation 1:1 zwischen Rivaroxaban (20 mg/Tag Abendeinnahme empfohlen) und VKA (INR zwischen 2.0 und 3.0) randomisiert. Die OAK wurde 1-7 Tage (dann war ein Thrombenausschluss mit transösophagealer Echokardiographie oder intrakardialem Ultraschall erforderlich) oder dokumentiert für durchgehend 3 Wochen vor der Intervention begonnen. Die letzte Gabe von Rivaroxaban erfolgte (präferenziell am Abend) am Tag vor der Ablation und die erste Gabe danach 6-12h nach Erreichen der Hämostase. Während der Intervention wurde unfraktioniertes Heparin (mit einem ACT Zielwert von 300-400 Sek) verabreicht. Die OAK-Therapie wurde über mindestens 4 Wochen nach dem Eingriff gegeben. In beiden Therapiearmen waren die Raten der Blutungs- und ThrombembolieEreignisse niedrig und vergleichbar, d.h. Rivaroxaban den VKA nicht unterlegen.

In Hinblick auf die Verwendung von niedrigeren Rivaroxaban-Dosen (15 mg/Tag) oder die Verhinderung von stummen zerebralen Ischämien (nachgewiesen mittels prä- und post-interventionellem MRI) liegen 
keine Daten aus randomisierten Studien zwischen Rivaroxaban und VKAs vor.

\section{Apixaban}

Die Überlegenheit von Apixaban in der Thrombembolie-Prophylaxe im Vergleich zu VKA bei Patienten mit nicht-valvulärem VHF wurde in ARISTOTLE nachgewiesen [53]. Innerhalb von 1,8 Jahren Follow-up konnten in dieser Studie das Auftreten eines Schlaganfalls oder einer Thrombemolie relativ um $21 \%$ (1,27\% vs. $1,6 \%)$ und das von schweren Blutungen relativ um $31 \%(2,13 \%$ vs. 3,09\%) reduziert werden. Im Gegensatz zu anderen NOAC schlug sich dieser Vorteil auch in einer $10 \%$ igen relativen Risikoreduktion in der Gesamtmortalität $(3,52 \%$ vs. $3,94 \%)$ der Patienten unter $2 \times 5 \mathrm{mg}$ Apixaban nieder. Der Netto-Benefit der neuen Substanz konnte auch in retrospektiven Analysen von großen nationalen Datenbanken gezeigt werden und ist daher mit großer Wahrscheinlichkeit auch auf die Patienten der täglichen Praxis übertragbar [54].

Apixaban wird auch bei Patienten angewandt, die sich einer PVI unterziehen lassen. Eine Reduktion von Thrombembolien ohne eine Zunahme von Blutungen konnte schon in monozentrischen, kleinen Studien und einem prospektiven Register gezeigt werden [21-23]. Auch eine Metaanalyse, in der 4 Studien und insgesamt fast 1700 Patienten zusammengefasst wurden, konnte keine signifikanten Unterschiede zwischen Apixaban und VKA finden, wenn die beiden Medikamente jeweils ohne Unterbrechung im Rahmen einer PVI appliziert wurden [24]. Eine zweite Metaanalyse kam zu dem Resultat, dass Apixaban im Rahmen der PVI ähnlich effektiv in der Verhinderung thrombembolischer Ereignisse bei gleichzeitig überlegener Sicherheit bezüglich schwerer Blutungen ist [25]. Auch hinsichtlich der SZL zeigte sich in einer (sehr kleinen) Kohorte ein günstiger Effekt: Eine cerebrale MRT am Tag nach der Ablation wurde in dieser Publikation zwar nur bei 29 Patienten im Apixaban-Arm durchgeführt, Pathologien fanden sich jedoch bei keinem einzigen Patienten [23].

In der AEIOU Studie wurde Apixaban in zwei verschiedenen Dosierungen untersucht und am HRS Kongress in Chicago im Mai 2017 präsentiert. Apixaban, ununterbrochen oder unterbrochen, zeigte eine ähnliche Effektivität wie ein ununterbrochener VKA in der Verhinderung von Schlaganfall und systemischer Thrombembolie. Auch die Blutungsraten zeigten sich vergleichbar mit insbesondere wenigen schweren Blutungen. Ohne der Vollpublikation dieser Studie vorgreifen $\mathrm{zu}$ wollen, kann aus den bisherigen Ergebnissen wohl abgeleitet werden, dass eine Therapie mit Apixaban im Rahmen einer PVI ununterbrochen oder einmal unterbrochen (Pausieren der Morgendosis vor Ablation) effektiv und sicher ist [20].

Die ununterbrochene Apixaban-Therapie im Rahmen einer PVI wird derzeit in der randomisierten, prospektiven und multizentrischen AXAFA-AFNET-
5 Studie untersucht [55]. In diesem Protokoll, bei dem auch 4 Österreichische Zentren beteiligt sind, werden ca. 650 Patienten entweder mit VKA oder mit Apixaban 1 Monat vor bis 3 Monate nach einer PVI verglichen. Neben der Rate an neurologischen Defiziten und Blutungen unter den Substanzen wird auch das Auftreten von SZL in der MRT am Tag nach der Ablation untersucht. Erste Ergebnisse wurden im März 2018 (EHRA meeting) als late breaking clinical trial angekündigt.

Ohne die Ergebnisse dieser wichtigen Studie vorzugreifen, kann aus den bisherigen Ergebnissen wohl abgeleitet werden, dass eine Therapie mit Apixaban im Rahmen einer PVI nicht unterbrochen werden muss bzw. sollte.

\section{Edoxaban}

Mit Edoxaban ist seit 2017 der bisher dritte Faktor Xa Inhibitor am Europäischen Markt verfügbar. Die Sicherheit und Wirksamkeit dieser Substanz bei Patienten mit VHF im Vergleich mit Warfarin wurde in der ENGAGE AF-TIMI 48 Studie nachgewiesen [26]. In eine Subgruppen-Analyse dieser großen randomisierten Studie wurden 169 Patienten eingeschlossen, die einer PVI unterzogen wurden [56]. Innerhalb von 30 Tagen nach dem durchgeführten Eingriff wurde ein ischämischer Schlaganfall in der VKA Gruppe im Vergleich zu keinem Ereignis unter einer der beiden Edoxaban-Dosierungen verzeichnet. Hinsichtlich der Blutungen war Edoxaban der Kontrollgruppe numerisch überlegen. So wurden drei relevante Blutungen in der VKA, aber nur eine schwere Blutung unter $60 \mathrm{mg}$ und eine leichte Blutung unter $30 \mathrm{mg}$ Edoxaban beschrieben. Als Einschränkung dieser Analyse ist jedoch anzumerken, dass die Fallzahlen sehr klein waren und die Dauer der Unterbrechung der Studienmedikation den behandelten Ärzten freigestellt wurde. Hinsichtlich genauer Daten zur Verwendung von Edoxaban während einer PVI werden die Ergebnisse der ELIMINATE-AF Studie für 2019 erwartet. Bei dieser Studie wird der VKA oder $30 \mathrm{mg} / 60 \mathrm{mg}$ Edoxaban ohne Unterbrechung während der PVI randomisiert verglichen.

Kontroversiell ist bisher das Management bei Auftreten einer schweren Blutung unter allen Faktor-Xa Inhibitoren, insbesondere bei einer Perikardtamponade oder einer nötigen (z. B. vaskulären) Akut-Operation. Nachdem für die Faktor-Xa Inhibitoren noch kein direktes Antidot im Handel ist, müssen diese Patienten mangels Alternativen mit Frischplasma oder Gerinnungsfaktoren behandelt werden. Die klinische Anwendung des ersten Antidots (Andexanet Alfa) ist derzeit in Erprobung, die Einführung im Europäischen Markt ist allerdings nicht vor 2018 zu erwarten [57]. 


\section{Zusammenfassung}

Die Consensus-Empfehlungen werden in Tab. 1 dargestellt. Grundsätzlich sollte für die OAK während der PVI ein institutionelles Vorgehen vorliegen, das auf der aktuellen wissenschaftlichen Datenlage und den Erfahrungen des jeweiligen Zentrums mit der Antikoagulation beruht.

Zusammenfassend kann, mit unterschiedlichen Empfehlungsklassen und Evidenzgraden, die ununterbrochene OAK im Rahmen der PVI, sowohl für VKA als auch für sämtliche derzeit verfügbare NOAC (inklusive morgentliche Gabe), empfohlen werden. Es kann bei NOAC ebenso eine zeitliche Unterbrechung mit Pausieren der morgendlichen Gabe und einem Neustart erfolgen. Ein Switch von NOAC auf VKA vor Ablation ist prinzipiell möglich. Für ein individuelles Vorgehen können modifizierende Faktoren miteinbezogen werden, die das Intervall der Unterbrechung mitbestimmen: Nierenfunktion, $\mathrm{CHA}_{2} \mathrm{DS}_{2}$ VASc-Score, Erfahrung in der transseptalen Punktion, zusätzliche Bildgebung zur transseptalen Punktion (intrakardialer Ultraschall/transösophageales Echo), geplante zusätzliche Ablation zur PVI. Auch bei niedrigem $\mathrm{CHA}_{2} \mathrm{DS}_{2}$-VASc-Score ist eine OAK 3-4 Wochen vor dem Eingriff indiziert, alternativ ist ein Ausschluss intraatrialer Thromben mittels transösophagealer Echokardiographie möglich. Eine OAK ist zumindest 8 Wochen nach dem Eingriff indiziert und danach vom Risikoprofil des Patienten, nach dem $\mathrm{CHA}_{2} \mathrm{DS}_{2}$-VASc-Score abhängig. Periinterventionelles Bridging mit Heparinen sollte aufgrund der vermehrten Blutungsereignisse vermieden werden.

Der vorliegende Consensus stellt Empfehlungen nach dem derzeitigen Wissensstand dar und wurde ausschließlich von Mitgliedern der Arbeitsgruppe Rhythmologie der Österreichischen Kardiologischen Gesellschaft erstellt, welche große praktische Erfahrung in der Katheterablation und periinterventionellen OAK bei Patienten mit VHF besitzen. Es ist in den nächsten Monaten die Veröffentlichung neuer randomisierter und kontrollierter Studien zum vorliegenden Thema zu erwarten, sodass es sicherlich zu Änderungen in den Empfehlungen kommen wird. Die AG-Rhythmologie der ÖKG wird sich bemühen, diese S1-Leitlinie regelmäßig auf dem neuesten Stand zu halten. Wir hoffen, durch diese Leitlinie die Sicherheit der Patienten, die einer PVI unterzogen werden, zu erhöhen und für die behandelnden Ärzte, ein österreichweit möglichst homogenes Vorgehen zu ermöglichen.

Funding Open access funding provided by Medical University of Vienna.

Interessenkonflikt M. Martinek: Vortragshonorare, Mitglied bei Advisory Boards, Fortbildungsunterstützungen von Boehringer-Ingelheim, Bayer, BMS, Pfizer. M. Gwechenberger: Vortragshonorare, Mitglied bei Advisory Boards, Fortbildungsunterstützungen von Boehringer-Ingelheim, Bayer,
Daichii, BMS. D. Scherr: Vortragshonorare von Boehringer-Ingelheim, Bayer, BMS, Daiichi. C. Steinwender: Vortragshonorare Boehringer-Ingelheim, Bayer, Daiichi-Sankyo. M. Stühlinger: Vortragshonorare Boehringer-Ingelheim, Bayer, BMS, Pfizer, Daiichi-Sankyo. H. Pürerfellner: Vortragshonorare oder Honoraria von Bayer, Daiichi-Sankyo, Boehringer-Ingelheim, Pfizer, Abbott, Biosense-Webster, Boston Scientific, Medtronic. F.X. Roithinger: Vortragshonorare, Mitglied bei Advisory Boards, Fortbildungsunterstützungen von Boehringer-Ingelheim, Bayer, BMS, Abbott, Daiichi-Sankyo, Pfizer, Biosense-Webster, Boston Scientific, Medtronic. L. Fiedler: Vortragshonorare, Mitglied bei Advisory Boards, Fortbildungsunterstützungen von BoehringerIngelheim, Bayer, BMS, Abbott.

Open Access Dieser Artikel wird unter der Creative Commons Namensnennung 4.0 International Lizenz (http:// creativecommons.org/licenses/by/4.0/deed.de) veröffentlicht, welche die Nutzung, Vervielfältigung, Bearbeitung, Verbreitung und Wiedergabe in jeglichem Medium und Format erlaubt, sofern Sie den/die ursprünglichen Autor(en) und die Quelle ordnungsgemäß nennen, einen Link zur Creative Commons Lizenz beifügen und angeben, ob Änderungen vorgenommen wurden.

\section{Literatur}

1. Calkins H, et al. 2017 HRS/EHRA/ECAS/APHRS/SOLAECE Expert Consensus Statement on Catheter and Surgical Ablation of Atrial Fibrillation. Heart Rhythm Journal. 2017;14,10:e275:e444.

2. Kirchhof P, et al. Issue 38, 7 October 2016. ESC Guidelines for the management of atrial fibrillation developed in collaboration with EACTS. European Heart Journal, Vol. 37. 2016.pp. 2893-962.

3. Calkins H, Kuck KH, Cappato R, Brugada J, Camm AJ, Chen SA, et al. HRS/EHRA/ECAS Expert Consensus Statement on Catheter and Surgical Ablation of Atrial Fibrillation: recommendations for patient selection, procedural techniques, patient management and follow-up, definitions, endpoints, and research trial design. Europace. 2012;2012(14):528-606.

4. Cappato R, Calkins H, Chen SA, Davies W, IesakaY, Kalman J, et al. Updated world-wide survey on the methods, efficacy, and safety of catheter ablation for human atrial fibrillation. CircArrhythm Electrophysiol. 2010;3:32-8.

5. Calkins $\mathrm{H}$, et al. HRS/EHRA/ECAS. recommendations for personnel, policy, procedures and follow-up. A report of the Heart Rhythm Society (HRS) Task Force on catheter and surgical ablation of atrial fibrillation. Heart Rhythm: expert Consensus Statement on catheter and surgical ablation of atrial fibrillation; 2007.4(6):816-61.

6. Camm AJ, Lip GY, De Caterina R, Savelieva I, Atar D, HohnloserSH, etal.ESCCommitteeforPracticeGuidelinesCPG; Document Reviewers. 2012 Focused up- date of the ESC Guidelines for the management of atrial fibrillation: an update of the 2010 ESC Guidelines for the management of atrial fibrillation - developed with the special contribution of the European Heart Rhythm Association. Europace. 2012;14:1385-413.

7. Santangeli P, Di Biase L, Horton R, Burkhardt JD, Sanchez J, Al-Ahmad A, et al. Ablation of atrial fibrillation under therapeutic warfarin reduces periprocedural com - plications: evidence from a meta - analysis. Circ Arrhythm Electrophysiol. 2012;5:302-11.

8. Di Biase L, Burkhardt JD, Santangeli P, Mohanty P, Sanchez JE, Horton R, et al. Peri- procedural stroke and bleeding 
complications in patients undergoing catheter ablation of atrial fibrillation with different anticoagulation management: results from the Role of Coumadin in Preventing Thromboembolism in Atrial Fibrillation (AF) Patients Undergoing Catheter Ablation (COMPARE) randomized trial. Circulation. 2014;129:2638-44.

9. Calkins H, Willems S. Gerstenfeld EP et al. RE-CIRCUIT Investigators. NEnglJ Med. 2017;27;376(17):1627-36. Apr.

10. Glund S, Moschetti V, Norris S, et al. A randomised study in healthy volunteers to investigate the safety, tolerability and pharmacokinetics of idarucizumab, a specific antidote to dabigatran. Thromb Haemost. 2015;113:943-51.

11. Glund S, Stangier J, van Ryn J, et al. Effect of Age and Renal Function on Idarucizumab Pharmacokinetics and Idarucizumab-Mediated Reversal of Dabigatran Anticoagulant Activity in a Randomized, Double-Blind, Crossover Phase IbStudy. Clin Pharmacokinet. 2017;56(1):41-54.

12. Pollack CV Jr, Reilly PA, Eikelboom J, et al. Idarucizumab for dabigatran reversal. NEngl J Med. 2015;373:511-20.

13. Pollack CV Jr, Reilly PA, van Ryn J, et al. Idarucizumab for Dabigatran Reversal-Full Cohort Analysis. N Engl J Med. 2017;3;377(5):431-41. Aug.

14. Kaiser DW, Streur MM, Nagarakanti R, Whalen SP, Ellis CR. Continuous warfarin versus periprocedural dabigatran to reduce stroke and systemic embolism in patients undergoing catheter ablation for atrial fibrillation or left atrial flutter.J Interv Card Electrophysiol. 2013;37:241-7.

15. Nagao T, Inden Y, Shimano M, et al. Feasibility and safety of uninterrupted dabigatran therapy in patients undergoing ablation for atrial fibrillation. Intern Med. 2015;54:1167-73.

16. Maddox W, Kay GN, Yamada T, et al. Dabigatran versus warfarin therapy for uninterrupted oral anticoagulation during atrial fibrillation ablation. J Cardiovasc Electrophysiol. 2013;24:861-5.

17. Zhao Y, Yang Y, Tang X, Yu X, Zhang L, Xiao H. New oral anticoagulants compared to warfarin for perioperative anticoagulation in patients undergoing atrial fibrillation catheter ablation: a metaanalysis of continuous or interrupted new oral anticoagulants during ablation compared to interrupted or continuous warfarin. J Interv Card Electrophysiol. 2017;48(3):267-82.

18. Cappato R, Marchlinski F, Hohnloser SH, et al. Uninterrupted rivaroxaban vs. uninterrupted vitamin $\mathrm{K}$ antagonists for catheter ablation in non-valvular atrial fibrillation. Eur Heart J.2015;36:1805-11.

19. Vamos M, Cappato R, Marchlinski FE, Natale A, Hohnloser SH. Efficacy and safety of rivaroxaban compared with vitamin $\mathrm{K}$ antagonists for peri-procedural anticoagulation in catheter ablation of atrial fibrillation: a systematic review and meta-analysis. Europace. 2016;18:1787-94.

20. Reynolds MR. LBCT01. Heart RhythmJSuppl2017;May:10 13

21. Kuwahara T, et al. Apixaban versus Warfarin for the Prevention ofPeriproceduralCerebralThromboembolisminAtrial Fibrillation Ablation: Multicenter Prospective Randomized Study. J Cardiovasc Electrophysiol. 2016;27(5):549-54.

22. Shah RR, et al. Safety and Efficacy of Uninterrupted Apixaban Therapy versus Warfarin During Atrial Fibrillation Ablation. Am JCardiol. 2017;120(3):404-7.

23. Di Biase L, et al. Feasibility and safety of uninterrupted periprocedural apixaban administration in patients undergoing radiofrequency catheter ablation for atrial fibrillation: Results from a multicenter study. Heart Rhythm. 2015;12(6):1162-8.

24. Blandino A, et al. Apixaban for periprocedural anticoagulation during catheter ablation of atrial fibrillation: a sys- tematic review and meta-analysis of 1691 patients. J Interv Card Electrophysiol.2016;46(3):p:225-36.

25. BaiY, ShiXB, MaCS, Lip GYH. Meta-Analysis of Effectiveness and Safety of Oral Anticoagulants in Atrial Fibrillation With Focus on Apixaban. Am J Cardiol. 2017;120(9):1689-1695. https://doi.org/10.1016/j.amjcard.2017.07.072. Epub 2017 Aug 1.

26. Giugliano RP, et al. Edoxaban versus warfarin in patients with atrialfibrillation. NEnglJ Med.2013;369(22):2093-104.

27. Wazni OM, et al. Embolic events and char formation during pulmonary vein isolation in patients with atrial fibrillation: impact of different anticoagulation regimens and importance of intracardiac echo imaging. J Cardiovasc Electrophysiol.2005;16(6):576-81.

28. Schmidt M, et al. Atrial fibrillation ablation in patients with therapeutic international normalized ratios. Pacing Clin Electrophysiol.2009;32(8):995-9.

29. Martinek M, Sigmund E, Lemes C, et al. Asymptomatic cerebrallesions during pulmonaryveinisolation under uninterrupted oral anticoagulation. Europace. 2013;15(3):325-31. Mar.

30. Bassiouny $\mathrm{M}$, et al. Use of dabigatran for periprocedural anticoagulation in patients undergoing catheter ablation for atrial fibrillation. Circ Arrhythm Electrophysiol. 2013;6(3):460-6.

31. Bin Abdulhak AA, et al. Safety and efficacy of interrupted dabigatran for peri-procedural anticoagulation in catheter ablation of atrial fibrillation: a systematic review and metaanalysis. Europace. 2013;15(10):1412-20.

32. Hohnloser SH, Camm AJ. Safety and efficacy of dabigatran etexilate during catheter ablation of atrial fibrillation: a meta-analysis of theliterature.Europace.2013;15(10):1407-11.

33. Providencia R, et al. Rivaroxaban and dabigatran in patients undergoing catheter ablation of atrial fibrillation. Europace. 2014;16(8):1137-44.

34. Winkle RA, et al. Peri-procedural interrupted oral anticoagulation for atrial fibrillation ablation: comparison of aspirin, warfarin, dabigatran, and rivaroxaban. Europace. 2014;16(10):1443-9.

35. Armbruster HL, et al. Safety of novel oral anticoagulants compared with uninterrupted warfarin for catheter ablation of atrial fibrillation. Ann Pharmacother. 2015;49(3):278-84.

36. January CT, et al. 2014 AHA/ACC/HRS guideline for the management of patients with atrial fibrillation: a report of the American College of Cardiology/American Heart Association Task Force on Practice Guidelines and the Heart Rhythm Society. JAm Coll Cardiol. 2014;64(21):e1-76.

37. Nairooz R, Ayoub K, Sardar P, et al. Uninterrupted New Oral Anticoagulants Compared With Uninterrupted Vitamin $\mathrm{K}$ Antagonists in Ablation of Atrial Fibrillation: A Metaanalysis. Can J Cardiol.2016;32:814-23. Jun.

38. Wu S, Yang YM, Zhu J, et al. Meta-Analysis of Efficacy and Safety of New Oral Anticoagulants Compared With Uninterrupted Vitamin K Antagonists in Patients Undergoing Catheter Ablation for Atrial Fibrillation. Am J Cardiol. 2016;117:926-34.

39. Connolly SJ, Ezekowitz MD, YusufS, et al. Dabigatran versus warfarin in patients with atrial fibrillation. N Engl J Med. 2009;361:1139-51.

40. Eikelboom JW, Wallentin L, Connolly SJ, et al. Risk of bleeding with 2 doses of dabigatran compared with warfarin in older and younger patients with atrial fibrillation: an analysis of the Randomized Evaluation of Long-Term Anticoagulant Therapy (RE-LY) trial. Circulation. 2011;123:2363-72.

41. Romanelli RJ, Nolting L, Dolginsky M, Kym E, Orrico KB. Dabigatranversuswarfarinforatrialfibrillationin realworld 
clinical practice: a systematic review and meta-analysis. Circ Cardiovasc Qual Outcomes. 2016;9:126-34.

42. Larsen TB, Rasmussen LH, Skjøth F, et al. Efficacy and safety of dabigatran etexilate and warfarin in „real-world“ patients with atrial fibrillation: a prospective nationwide cohort study. JAm Coll Cardiol. 2013;61:2264-73.

43. GrahamDJ, Reichman ME, Wernecke M, etal. Stroke, bleeding, and mortality risks in elderly Medicare beneficiaries treated with dabigatran or rivaroxaban for nonvalvular atrial fibrillation. Jama Intern Med. 2016;176:1662-71.

44. Graham DJ, Reichman ME, Wernecke M, et al. Cardiovascular, bleeding, and mortality risks in elderly Medicare patients treated with dabigatran or warfarin for nonvalvular atrial fibrillation. Circulation. 2015;131:157-64.

45. Kim JS, She F, Jongnarangsin K, et al. Dabigatran vs warfarin for radiofrequency catheter ablation of atrial fibrillation. Heart Rhythm. 2013;10:483-9.

46. Yamaji H, Murakami T, Hina K, et al. Usefulness of dabigatran etexilate as periprocedural anticoagulation therapy for atrial fibrillation ablation. Clin Drug Investig. 2013;33:409-18.

47. Bassiouny M, Saliba W, Rickard J, et al. Use of dabigatran for periprocedural anticoagulation in patients undergoing catheter ablation for atrial fibrillation. Circ Arrhythm Electrophysiol. 2013;6:460-6.

48. Nin T, Sairaku A, Yoshida Y, et al. A randomized controlled trial of dabigatran versus warfarin for periablation anticoagulation in patients undergoing ablation of atrial fibrillation. Pacing Clin Electrophysiol. 2013;36:172-9.

49. Stepanyan G, Badhwar N, Lee RJ, et al. Safety of new oral anticoagulants for patients undergoing atrial fibrillation ablation. J Interv Card Electrophysiol. 2014;40:33-8.
50. Arshad A, Johnson CK, Mittal S, et al. Comparative safety of periablation anticoagulation strategies for atrial fibrillation: data from a large multicenter study. Pacing Clin Electrophysiol. 2014;37:66573.

51. Armbruster HL, Lindsley JP, Moranville MP, et al. Safety of novel oral anticoagulants compared with uninterrupted warfarin for catheter ablation of atrial fibrillation. Ann Pharmacother. 2015;49:278-84.

52. Patel MR, Mahaffey KW, Garg J, Pan G, Singer DE, Hacke $\mathrm{W}$, et al. Rivaroxaban versus warfarin in nonvalvular atrial fibrillation. NEngl J Med. 2011;365:883-91.

53. Granger CB, et al. Apixaban versus warfarin in patients with atrial fibrillation. NEngl J Med. 2011;365(11):981-92.

54. Li XS, et al. Effectiveness and safety of apixaban versus warfarin in non-valvular atrial fibrillation patients in ,realworld" clinical practice. A propensity-matched analysis of 76,940 patients. Thromb Haemost. 2017;117(6):1072-82.

55. Di Biase L, et al. Rationale and design of AXAFA-AFNET 5: an investigator-initiated, randomized, open, blinded outcome assessment, multi-centre trial to comparing continuous apixaban to vitamin $\mathrm{K}$ antagonists in patients undergoing atrial fibrillation catheter ablation. Europace. 2017;19(1):132-8.

56. Steffel J, et al. First experience with edoxaban and atrial fibrillation ablation - Insights from the ENGAGE AF-TIMI 48 trial. Int J Cardiol. 2017;244:192-195. https://doi.org/10. 1016/j.ijcard.2017.05.098. Epub2017 Jun 2.

57. Connolly SJ, et al. Andexanet Alfa for Acute Major Bleeding Associated with Factor Xa Inhibitors. N Engl J Med. 2016;375(12):1131-41. 